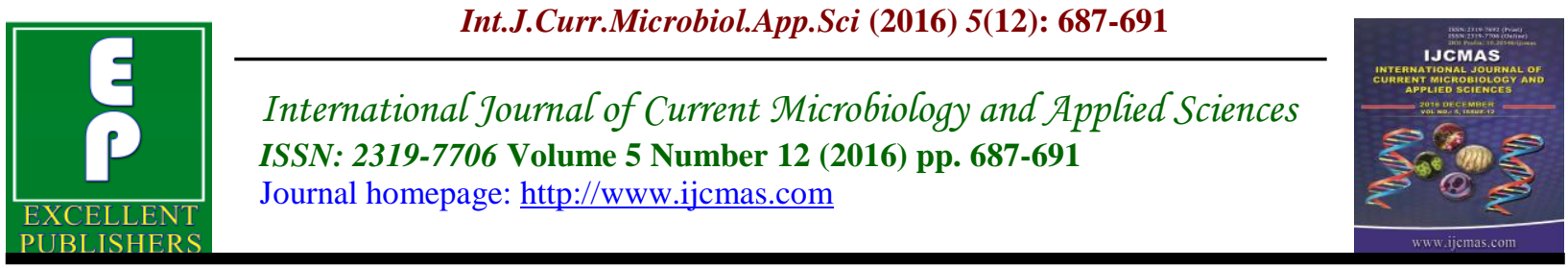

Original Research Article

http://dx.doi.org/10.20546/ijcmas.2016.512.077

\title{
A Study on Aetiology of Onychomycosis
}

\author{
G. Jeena and N. Saritha* \\ Department of Microbiology, Government Medical College, \\ Thiruvananthapuram 695011, Kerala, India \\ *Corresponding author
}

\section{Keywords}

Onychomycosis, Dermatophytes and non dermatophytes, Candida albicans.

Article Info

Accepted:

26 November 2016 Available Online:

10 December 2016

\section{A B S T R A C T}

Onychomycosis is probably the most common of all nail disorders, accounting for approximately $50 \%$ of all nail problems. Due to the increasing incidence of onychomycosis, it was thought worthwhile to undertake a clinicomycological study on this problem. 100 Patients seeking medical advice at the Dermatology outpatient department for nail abnormalities and who had clinical features suggestive of onychomycosis were included in the study for a period of one year. Scrapings were collected from the advancing infected edge as close to the cuticle as possible and from the underside of nail plate with a sterile scalpel blade, and direct microscopy using $10 \% \mathrm{KOH}$ and culture on SDA was done. The tubes were incubated at room temperature for four weeks. Dermatophytes and non dermatophytes were identified by gross colony morphology, microscopic examination with Lactophenol cotton blue (LCB) preparation and by gram staining and Candida albicans was confirmed by germ tube test. Direct microscopy was positive in 57 patients while 43 patients did not yield a positive result. Culture positivity was noted in 51 patients. Trichophyton species was the most common pathogen isolated in cultures $(45.09 \%)$. Among these 12 patients yielded T.rubrum, 9 yielded T.mentagrophytes and 19 yielded Candida albicans. Direct microscopy using $\mathrm{KOH}$ is a simple and relatively quick method to detect the presence of fungus. Culture helps to identify the species of the fungus.

\section{Introduction}

Onychomycosis denotes any infection of the nail caused by dermatophyte fungi, non dermatophyte fungi or yeasts. Tinea unguim, refers strictly to the dermatophyte infection of the nail plate (Nelson et al., 2003; Clayton, 1992).

Onychomycosis is probably the most common of all nail disorders, accounting for approximately $50 \%$ of all nail problems (Scher, 1994). They occur worldwide, but with variable frequency depending on the climatic, professional and socioeconomic conditions (Baran et al., 2006). In recent years there appears to have been a substantial increase in number of patients suffering from mycotic nail disorders. The increasing use of systemic antibiotics, immunosuppressive agents, invasive surgical procedures and organ transplants may be responsible for this situation. Dermatophytes are the most frequently 
implicated causative agents in onychomycosis. They are able to invade normal keratin. Previously regarded as contaminants, yeasts are also now increasingly recognised as pathogens, as are some moulds. As onychomycosis is responsible for only $50 \%$ of dystrophic nails, laboratory confirmation is often helpful prior to treatment with an oral antifungal. Potassium hydroxide examination $(\mathrm{KOH})$, nail biopsy and fungal culture are most useful.

Due to the increasing incidence of onychomycosis, it was thought worthwhile to undertake a clinicomycological study on this problem.

The main aim of this study, to identify the species of fungal aetiological agent. And also to compare the clinical diagnosis with $\mathrm{KOH}$ mount positivity and culture positivity

\section{Materials and Methods}

Type of study : Descriptive study

Place of study: Departments of Dermatology and Venereology and Microbiology, Government Medical College, Thiruvananthapuram, Kerala, India

Duration of study: One year

Inclusion criteria: All patients attending Dermatology outpatient department with clinical features of onychomycosis

Sample size: fixed at 80 using the formula $4 \mathrm{pq} / \mathrm{d} 2$

Where $\mathrm{p}$ - prevalence of $\mathrm{KOH}$ and/or culture positivity among clinically diagnosed cases of onychomycosis fixed at 54.5.

$q-100-p$ and $d-20 \%$ of $p$

\section{Methodology}

Patients seeking medical advice at the Dermatology outpatient department for nail abnormalities and who had clinical features suggestive of onychomycosis were included in the study. The study was conducted over aperiod of one year from December 2007 to November 2008.

After getting an informed consent, a detailed history with particular emphasis on occupation, history of trauma, prolonged contact with water, chronic paronychia and associated disorders like diabetes mellitus was taken with the help of preset proforma.

A detailed clinical examination, dermatological examination and systemic examination was done in each patient. Complete hemogram, urine examination and blood sugar level estimation was done in each patient.

Specimen was collected from the affected nails after cleaning the nail and surrounding skin with spirit to remove any contaminants. Scrapings were collected from the advancing infected edge as close to the cuticle as possible and from the underside of nail plate with a sterile scalpel blade, Separate specimens were taken from each pattern if a patient had more than one pattern of nail involvement.

\section{Direct Microscopy}

Direct microscopic examination of the material was performed after keeping it in $10 \% \mathrm{KOH}$ for 24 hours. The alkaline clearing solution will digest the proteins, lipids and epithelial debris, but fungal elements are resistant because of their chitinous cell wall. In a positive preparation, the fungi appear as highly refractile septate branching hyphal elements. Artefacts 
include fibres of cotton, starch grains, fat droplets and mosaic fungus.

The rest of the specimen was cultured in Sabourauds dextrose agar (SDA) with and without cycloheximide and chloramphenicol. Specimens were inoculated into the culture medium with a sterile loop or scalpel by furrowing into the medium as much of the material as possible. The tubes were incubated at room temperature for four weeks. Culture was examined daily for colony growth. Dermatophytes and non dermatophytes were identified by gross colony morphology, microscopic examination with Lactophenol cotton blue (LCB) preparation and by gram staining and Candida albicans was confirmed by germ tube test. Lack of growth for 4 weeks was considered culture negative. If a dermatphyte was isolated in culture, it was taken as pathogen and if nondermatophyte grows, repeated culture positivity is required to consider it as pathogen.

\section{Statistical Analysis}

Data was analysed using SPSS version 10. The data was expressed in frequency and percentage. Chi square test was used to find out the statistical association between two qualitative variables. The risk was measured using Odds ratio with its $95 \%$ confidence limits. Kappa was used to measure the agreement between two diagnostic tools (direct microscopy using $\mathrm{KOH}$ and fungal culture)

\section{Results and Discussion}

Even though, sample size was fixed as 80 , we could enrol 100 patients with onychomycosis in the study and the following observations were made.
The age of the patients ranged from 1 to 77 years and the maximum number of patients were in the age group 40-49 years(24\%). The maximum culture positivity was observed in age groups 30-39, 40-49 and 5059 years. The females were more in the study $(57 \%)$.

$78 \%$ of patients had symptoms for a duration of less than five years while only $5 \%$ had symptoms of duration more than 15 years. Occupational profile of the patients included in the study showed that the largest group consisted of homemakers (34\%).32 of the 66 patients who had culture positivity $(48.48 \%)$ gave a history of prolonged contact with water.

Diabetes mellitus as a comorbid condition was noted in $15 \%$ of patients included in the study. A history of dermatophytosis in other sites was present in $9 \%$ of patients, Tinea corporis in 5 patients and Tinea cruris in 4 patients. Paronychia was present in $51 \%$ patients while $49 \%$ had no paronychia.

Direct microscopy was positive in 57 patients while 43 patients did not yield a positive result. Culture positivity was noted in 51 patients. Among the 57 patients positive for direct microscopy, 42 were culture positive also. Out of the 51 patients who were culture positive, only $42(82.35 \%)$ showed direct microscopy positivity. But 15 patients with $\mathrm{KOH}$ positivity were culture negative.

Table 1 shows that the performance of the two diagnostic tools are significantly associated ( $\mathrm{p}$ value $=0.0000)$ and the measurement of agreement Kappa value 0.519 indicates that there is $51.9 \%$ agreement between direct microscopy and culture. 
Table.1 Correlation between direct microscopy and culture

\begin{tabular}{|l|l|l|l|}
\hline \multirow{2}{*}{ Direct Microscopy } & Culture & Total \\
\cline { 2 - 4 } & Positive & Negative & \\
\hline Positive & 42 & 15 & 57 \\
\hline Negative & 9 & 34 & 43 \\
\hline Total & 51 & 49 & 100 \\
\hline
\end{tabular}

Chi square value -27.295 , $\mathrm{p}$ value 0.000 , df:1, kappa -0.519

Out of the 51 culture positives, a total of 24 patients showed growth of dermatophytes $(47.05 \%)$. Of these, 12 isolates were Trichophyton rubrum, 9 were Trichophyton mentagrophytes, 2 were Trichophyton tonsurans and one was Epidermophyton. Candida albicans was the single largest group of pathogenic isolates with 19 (37.25\%) culture positives (Table 2).

Table.2 Frequency distribution of isolates in culture positive patients

\begin{tabular}{|l|c|}
\hline Isolates & Frequency and percentage \\
\hline T.rubrum & 12 \\
\hline T.mentagrophytes & 9 \\
\hline T.tonsurans & 2 \\
\hline E.floccosum & 1 \\
\hline Aspergillus niger & 6 \\
\hline Fusarium spp & 1 \\
\hline Acremonium spp & 1 \\
\hline Candida albicans & 19 \\
\hline Total & 51 \\
\hline
\end{tabular}

Trichophyton species was the most common pathogen isolated in cultures (45.09\%). Among these 12 patients yielded T.rubrum, 9 yielded T.mentagrophytes and 19 yielded Candida albicans.

The prevalence of onychomycosis has been reported to increase with advancing age possibly due to reduced rate of nail growth and increased trauma.16, 45. The maximum number of patients in this study was in the age group 40-49 years (24\%).This is in accordance with Gupta et al., (2007). The male: female ratio in this study was 1:1.32. This observation is in accordance with the studies by Lopes et al., Bokhari et al., and Sais et al., (1999, 1996). The predominance of female gender may be explained by their work habits; hands being wet most of the day and presence of aggravating factors like chemical detergents. The chronic indolent nature of the infection together with low therapeutic success explains the duration of complaints. Dogra et al., (2002) found that diabetics were 2.5 times more likely to have onychomycosis as observed in our study also. According to some authors, onychomycosis is no more common in diabetics than in general population (Rich, 1996).

Direct microscopy positivity rates and culture positivity rates in the present study is greater than that in studies by Shoar et al., (2002) but lower when compared to the studies by Sujatha et al., (2000). The most common isolate in our study was T.rubrum followed by $T$. mentagrophytes which is in accordance with other studies. Higher isolation rates of Candida albicans is in accordance with the study by Sujatha et al., (2006). 
In conclusion, onychomycosis is not an uncommon problem and its manifestations may be protean. Clinical examination plays an important role in suspecting a diagnosis of onychomycosis and determining the pattern and extent of nail involvement. Direct microscopy using $\mathrm{KOH}$ is a simple and relatively quick method to detect the presence of fungus. Culture helps to identify the species of the fungus. It is therefore advisable to get a laboratory confirmation using these methods for onychomycosis.

\section{References}

Baran, R., Hay, R., Haneke, E., Tosti, A. 2006. Onychomycosis: The current approach to diagnosis and therapy. $2^{\text {nd }}$ ed. UK: informa health care.

Bokhari, M.A., Hussain, I., Jahangir, M., Haroon, T.S., Aman, S., Khurshid, K. 1999. Onychomycosis in Lahore, Pakistan. Int. J. Dermatol., 38: 591-5.

Clayton, Y.M. 1992. Clinical and mycological diagnostic aspects of onychomycoses and dermetomycoses. Clin. Exp. Dermatol., 17(1): 37-40.

Dogra, S., Kumar, B., Bhansali, A., Chakrabarty, A. 2002. Epidemiology of onychomycosis in patients with diabetes mellitus in India. Int. J. Dermatol., 41: 647-51.

Gerami shoar, M., Zomorodian, K., Emami, M., Tarazoei, B., Saadat, F. 2002. Study and identification of the etiological agents of onychomycosis in Tehran, Iran. Iranian J. Public Health, 31(3-4): 100-104.

Grover, S. 2003. Clinico mycological evaluation of onychomycosis at Bangalore and Jorhat. Indian J. Dermatol. Venereol. Leprol., 69(4): 284-6.
Gupta, M., Sharma, N.L., Kanga, A.K., Mahajan, V.K., Tegta, G.R. 2007. Onychomycosis: Clinicomycologic study of 130 patients from Himachal Pradesh, India, Ind. J. Dermat. Vener Leprol., 73: 389-92.

Lopes, J.O., Alves, S.H., Mari, C.R., Oliveira, L.T., Brum, L.M., Westphalen, J.B., Furian, F.W., Altermann, M.J. 1999. A ten year survey of onychomycosis in the central region of the Rio Grande do Sul, Brazil. Rev. Inst. Med. Trop. Sao Paulo., 41(3) : 147-9.

Mercantini, R., Marsella, R., Morretto, D. 1996. Onychomycosis in Rome, Italy. Mycopathologia, 36: 25-32.

Nelson, M.M., Martin, A.G., Heffernan, M.P. 2003. Superficial fungal infection: Dermatophytosis, Onychomycosis, Tinea Nigra, Piedra. In: Freedberd IM, Eisen AZ, Wolff K, Austen KF, Goldsmith LA, Katz SI, editors. Fitzptricks Dermatology in General Medicine , $6^{\text {th }}$ ed. NewYork: Mc Graw hill, 205: p 1989-2005.

Rich, P. 2002. Special patient populations: Onychomycosis in the diabetic patient. $J$. Am. Acad. Dermatol., 35: S10-12.

Sais, G., Juggla, A., Peyri, J. 1996. Prevalence of dermatophyte onychomycosis in Rome, Italy. Mycopathologia, 6: 25-32.

Scher, R.K. 1994. Onychomycosis is more than a cosmetic problem. Br. J. Dermatol., 130(43): 15.

Sujatha, V., Grover, S., Dash, K., Singh, G. 2000. A clinic mycological evaluation of onychomycosis. Indian J. Dermatol. Venereol. Leprol., 66: 238-40.

Veer, P., Patwardhan, N.S., Damle, A.S. 2007. Study of onychomycosis: Prevailing fungi and pattern of infection. Indian J. Med. Microbiol., 25: 53-6.

\section{How to cite this article:}

Jeena, G., and Saritha, N. 2016. A Study on Aetiology of Onychomycosis. Int.J.Curr.Microbiol.App.Sci. 5(12): 687-691. doi: http://dx.doi.org/10.20546/ijcmas.2016.512.077 